\title{
TRAIL mutant membrane penetrating peptide alike (TMPPA) TRAIL-Mu3 enhances the antitumor effects of TRAIL in vitro and in vivo
}

\author{
HONG ZHU, JUAN YAN, QI XU, LIJIA WEI, XIANZHOU HUANG, SHOUCHUN CHEN and CHENG YI \\ Department of Medical Oncology, Cancer Center, West China Hospital, Sichuan University, \\ Chengdu, Sichuan 610041, P.R. China
}

Received February 24, 2017; Accepted September 6, 2017

DOI: $10.3892 / \mathrm{mmr} .2017 .7791$

\begin{abstract}
The aim of the present study was to prepare a tumor necrosis factor ligand superfamily member 10 (TRAIL) mutant membrane penetrating peptide alike (TMPPA), TRAIL-Mu3, and to investigate its antitumor effects in colorectal cancer in vitro and in vivo. The pMD19/TRAIL plasmid with designed primers was amplified to construct the target gene; it was ligated with an expression plasmid and the expression was confirmed. Subsequently, TRAIL-Mu3 was purified and further confirmed by western blot analysis. Immunofluorescence analysis was used to detect the distribution of TRAIL-Mu3 in colorectal cancer cells. In addition, the present study investigated the antitumor effects of TRAIL-Mu3 on colorectal cancer in vitro and in vivo. A novel TMPPA, TRAIL-Mu3, was synthesized in the present study. Following a series of detection experiments, it was confirmed that the TRAIL-Mu3 gene was obtained and was able to express TRAIL-Mu3 successfully. The immunofluorescence analysis demonstrated that TRAIL-Mu3 exhibited a markedly enhanced affinity to the colorectal cancer cell surface. In addition, TRAIL-Mu3 exerted stronger antitumor effects, compared with TRAIL, on colorectal cancer in vitro and in vivo.
\end{abstract}

\section{Introduction}

Tumor necrosis factor (TNF) ligand superfamily member 10 (TRAIL), a member of the TNF superfamily, is considered to

\footnotetext{
Correspondence to: Dr Shouchun Chen or Dr Cheng Yi, Department of Medical Oncology, Cancer Center, West China Hospital, Sichuan University, 3rd Inpatient Building, 37 Guo Xue Road, Chengdu, Sichuan 610041, P.R. China

E-mail: chen70257@126.com

E-mail: 1550930470@qq.com
}

Key words: tumor necrosis factor ligand superfamily member 10, trail mutant membrane penetrating peptide alike, colorectal cancer, antitumor effects be an optimal candidate for cancer therapy due to its tumor cell specificity and negligible cytotoxicity to normal cells in vitro and in vivo (1). TRAIL has antitumor activity in a variety of tumor cell lines (2,3). However, a large number of cancer cells, particularly in highly malignant tumors, are resistant to apoptosis induction by TRAIL, and cancer cells that were originally sensitive to TRAIL-induced apoptosis may become resistant following repeated exposure (4). Therefore, it is of importance to develop a more efficient form of TRAIL for cancer therapy.

Crossing biological barriers represents a principal limitation for the clinical application of biomolecules including nucleic acids, peptides or proteins (5). Previous studies have used cell-penetrating peptides (CPPs) as an efficient method for delivering therapeutic targets into cells $(6,7)$. CPPs, additionally termed protein transduction domains, comprise short and usually basic amino acid-rich peptides originating from proteins which are able to cross biological barriers. Typically, these peptides are cationic, rich in lysine or arginine residues, or amphipathic in nature $(8,9)$. CPPs have emerged as a novel class of non-viral vectors allowing for the delivery of various biomolecules across biological barriers from low molecular weight drugs to nano-sized particles (10).

In the present study, a number of amino acids of the N-terminal in soluble fragments (114-281aa) of the TRAIL protein were selectively altered to form penetrating peptide-like amino acid sequences. A total of $>10$ TRAIL penetrating peptide like mutants were synthesized. Following further detection and screening, one TRAIL penetrating peptide like mutant with eight consecutive Arg sequences, which approximately maintained the conformation of the TRAIL protein and had a penetrating peptide like structure, was identified. This TRAIL mutant membrane penetrating peptide alike (TMPPA) was termed TRAIL-Mu3. The present study examined the design, synthesis, expression, identification and purification of TRAIL-Mu3. In addition, the antitumor effects of TRAIL-Mu3 in colorectal cancer was detected in vitro and in vivo.

\section{Materials and methods}

Materials. The high-efficiency prokaryotic expression vector pET32a plasmid and the BL21 (DE3) competent 
Escherichia coli cells were purchased from Invitrogen (Thermo Fisher Scientific, Inc., Waltham, MA, USA). The pMD19-T vector was purchased from Takara Bio, Inc. (Otsu, Japan). The pMD19/TRAIL plasmid and wild-type TRAIL protein were obtained from Chengdu Huachuang Biotechnology Co., Ltd. (Chengdu, China). The TRAIL polyclonal antibody was purchased from Santa Cruz Biotechnology, Inc. (Dallas, TX, USA). The HCT-15, COLO 205, SW620, HT-29 and HCT 116 colon cancer cell lines were purchased from Wuhan Institute of Virology, Chinese Academy of Sciences (Wuhan, China). Female nude mice (6 weeks old) were purchased from the Animal Experimental Center of the Chinese Academy of Sciences (Shanghai, China).

Sequence and primer design of TRAIL-Mu3. The 114-121 amino acid coding sequence VRERGPQR of the wild-type TRAIL protein was selected and altered to become RRRRRRRR. The upstream primer (Mu3-TR-NdeI) was 5'-ggtcatatgcgtcgtcgtcgtcgtcgtcgtcgtgtggctgctcacatca-3' and the downstream primer (TR-EcoR) is 5'-gttgaattcttattaaccaac aaggaaagcaccgaagaaag-3'.

Amplification of the TRAIL-Mu3 gene. The genomic DNA of the pMD19/TRAIL plasmid was used as template DNA to perform polymerase chain reaction (PCR) analysis for the amplification of the TRAIL-Mu3 gene. Amplified products were purified and separated via electrophoresis (3\% agarose gel) to confirm whether the amplified products had the desired size (11).

Ligation of pMD19-T vector and TRAIL-Mu3 gene, and identification. The recovered TRAIL-Mu3 gene was ligated to the Takara pMD19-T vector and digested using EcoRI and HindIII enzymes. The gene was identified by electrophoresis. The plasmids of the corrected transformed bacteria containing the inserted gene segment were used for sequencing. The correctly sequenced bacteria were stored for further study (12).

Ligation of pET32a plasmid and TRAIL-Mu3 gene, and identification. The TRAIL-Mu3 DNA was ligated to the pET32a plasmid and digested by XbaI and EcoRI enzymes. Subsequently, the gene was identified by electrophoresis. The plasmids of the corrected transformed bacteria containing the inserted gene segment were used for sequencing. The correctly sequenced bacteria were stored for further study (12).

pET32a/TRAIL-Mu3 expression. The plasmid pET32a/TRAIL-Mu3 was transformed into BL21 (DE3) competent $E$. coli cells, which were cultured in Luria-Bertani solid medium containing Ampicillin at $4{ }^{\circ} \mathrm{C}$ overnight. A single colony was isolated and cultured. The cultured liquid was centrifuged $\left(13,800 \mathrm{x} \mathrm{g}, 4^{\circ} \mathrm{C}, 10 \mathrm{~min}\right)$ and the precipitate was resuspended to generate the prior-to-induction electrophoresis sample. The remaining liquid was cultured and induced with isopropyl $\beta$-D-thiogalactopyranoside to generate the post-induction electrophoresis sample.

The culture liquid was centrifuged $\left(13,800 \times \mathrm{g}, 4^{\circ} \mathrm{C}, 10 \mathrm{~min}\right)$ and the supernatant was discarded. The precipitate was resuspended, broken down using ultrasound and further centrifuged $\left(13,800 \times \mathrm{g}, 4^{\circ} \mathrm{C}, 10 \mathrm{~min}\right)$. The precipitate was resuspended.
The 20- $\mu 1$ supernatant and resuspended precipitate was used to make separate electrophoresis samples.

All the above electrophoresis samples were disposed in boiling water bath for $10 \mathrm{~min}$. Subsequently, the samples were centrifuged $\left(13,800 \mathrm{x} \mathrm{g}, 4^{\circ} \mathrm{C}, 10 \mathrm{~min}\right)$ and analyzed by electrophoresis (15\% gel, $200 \mathrm{~V}, 35 \mathrm{~min})$.

Purification of TRAIL-Mu3 protein. The TRAIL-Mu3 expression bacteria were broken down by ultrasound and centrifuged. The supernatant was removed and filtered with a $0.45-\mu \mathrm{m}$ membrane. Subsequently, the filtered supernatant was purified via cation exchange purification, hydroxyapatite purification and anion exchange purification methods, successively (13).

Western blot analysis of TRAIL-Mu3 protein. The TRAIL-Mu3 protein exhibited only 5 amino acids which were altered compared with the $\mathrm{N}$ terminal of the wild-type TRAIL protein, and the epitopes of the wild-type TRAIL protein were conserved. Therefore, a TRAIL polyclonal antibody was used for the detection and identification of TRAIL-Mu3 protein by western blotting $(14,15)$. The primary antibody used rabbit anti-human TRAIL polyclonal antibody (cat no. bs-1214R) (1:500) at $4^{\circ} \mathrm{C}$ overnight. The secondary antibody used was a goat anti-rabbit IgG-HRP (cat no. D2313; 1:5,000) at room temperature for $2 \mathrm{~h}$. Finally, the results were detected using an enhanced chemiluminescence detection reagent (Beyotime Institute of Biotechnology, Haimen, China).

Immunofluorescence analysis. The subcellular localization of TRAIL and TRAIL-Mu3 was detected in SW620 colon cancer cells by immunofluorescence (16).

TRAIL-Mu3-mediated toxicity in colorectal cancer cell lines. The antitumor effects of TRAIL-Mu3 and TRAIL were detected in 32 different tumor cell lines (including many TRAIL resistant tumor cell lines) through a Cell Counting Kit-8 (CCK-8). The cell growth inhibition rates of TRAIL-Mu3 and TRAIL in colorectal cancer cell lines (including HCT-15, COLO 205, SW620, HT-29 and HCT 116) measured by CCK-8 assay were discussed in this manuscript (17). The concentrations of reagents that induced a $50 \%$ reduction in cell viability [half-maximal inhibitory concentration, $\left(\mathrm{IC}_{50}\right)$ ] were determined from the curves of reagent concentration compared with the cell growth inhibition rate at $48 \mathrm{~h}$ of incubation for the cell line analyzed. The sensitivity of cells to a drug is evaluated by calculating the $\mathrm{IC}_{50}$ value; $\mathrm{IC}_{50}<10 \mu \mathrm{g} / \mathrm{ml}$ indicates that cells are sensitive to a drug, while $\mathrm{IC}_{50} \geq 10 \mu \mathrm{g} / \mathrm{ml}$ suggests that cells are relatively resistant to a drug (18-19).

Antitumor effects of HT-29 xenograft in nude mice. All animal procedures were approved by the Animal Care and Scientific Committee of Sichuan University (Chengdu, China). A total of 48 HT-29-bearing nude mice (SPF, aged 6-8 weeks, 18-22 g) were divided into 6 groups (8 mice/group): Group 1, treated with vehicle (saline); group 2, treated with TRAIL-Mu3 at a concentration of $5 \mathrm{mg} / \mathrm{kg}$; group 3, treated with TRAIL-Mu3 at a concentration of $15 \mathrm{mg} / \mathrm{kg}$; group 4, treated with TRAIL-Mu3 at a concentration of $45 \mathrm{mg} / \mathrm{kg}$; and group 5 , treated with TRAIL at a concentration of $45 \mathrm{mg} / \mathrm{kg}$. The mice were bred at $23 \pm 2^{\circ} \mathrm{C}$, with a humidity of $40-70 \%$ and a 


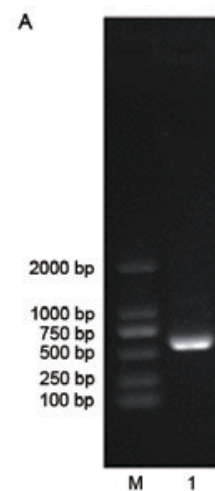

B

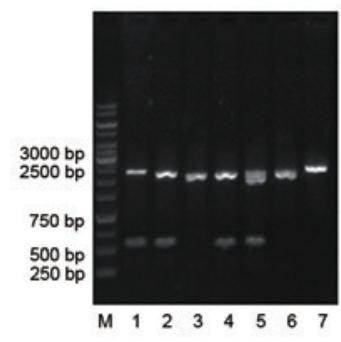

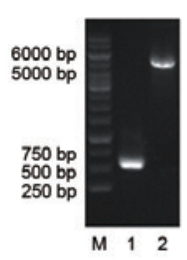

D

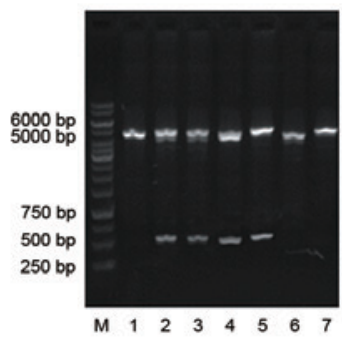

E

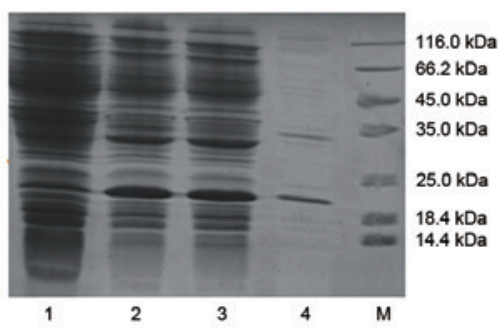

Figure 1. Results of electrophoresis identification during TRAIL-Mu3 synthesis. (A) Polymerase chain reaction products of the TRAIL-Mu3 gene. M, DNA marker; lane 1, TRAIL-Mu3. (B) Digestion of pMD19/TRAIL-Mu3 and electrophoresis products. M, DNA marker; lanes 1, 2, 4 and 5, four colonies transformed with pMD19/TRAIL-Mu3; lanes 3, 6 and 7, three colonies transformed with pMD19-T vector. (C) Digestion of the pET32a plasmid and TRAIL-Mu3 DNA. M, marker; lane 1, TRAIL-Mu3; lane 2,pET32a plasmid. (D) Digestion of pET32a/TRAIL-Mu3 and electrophoresis products. Lane M, marker; lanes 2, 3, 4 and 5, four colonies transformed with pET32a/TRAIL-Mu3; lanes 1, 6 and 7, three colonies transformed with the pET32a plasmid. (E) pET32a/TRAIL-Mu3 expression electrophoresis. Line M, marker; lane 1, induction electrophoresis sample; lane 2, post-electrophoresis sample; lane 3, supernatant; lane 4, precipitate. TRAIL, tumor necrosis factor ligand superfamily member 10 .

12/12 light/dark cycle with free access to food and water. The vehicle, TRAIL and TRAIL-Mu3 were injected through the tail vein five times in 5 days. The length, width, and weight of the tumor was measured using a slide caliper every 3 or 4 days. Tumor volume (TV) was estimated using the formula: TV $\left(\mathrm{mm}^{3}\right)=\left(\right.$ width $^{2} \mathrm{x}$ length $) / 2$. The growth inhibition rate of the tumor was calculated using the formula: Inhibition rate of tumor $(\%)=(1$ - average weight in treated group/average weight in control) x100 $(11,12)$.

Side effects. During the experimental period, side effects, including weight loss, mental state, appetite, behavior change and reactions, were observed.

Statistical analysis. The experiments were repeated at least three times. All data are expressed as the mean \pm standard error of the mean unless otherwise stated. Comparisons were analyzed using one-way analysis of variance and Least Significant Difference post hoc test. $\mathrm{P}<0.05$ was considered to indicate a statistically significant difference.

\section{Results}

PCR amplification of TRAIL-Mu3 gene. The amplified products of the TRAIL-Mu3 gene were detected by $3 \%$ agarose gel electrophoresis, and the results demonstrated that a specific segment of $\sim 500$ bp (Fig. 1A) was obtained.

Identification of the enzyme-digested products of pMD19/ TRAIL-Mu3. Following ligation of the pMD19-T vector and TRAIL-Mu3 genes, they were transformed into bacteria and cultured. A total of seven single colonies were isolated and the plasmids were extracted. Subsequently, the plasmids were digested and detected by agarose gel electrophoresis. The results demonstrated that there were two segments of $500 \mathrm{bp}$ and $\sim 2.7 \mathrm{~kb}$ in lines 1,2,4 and 5 (Fig. 1B), which were equal to the size of the TRAIL-Mu3 gene and pMD19-T vector plasmid, respectively. Therefore, these four colonies were transformed with pMD19/TRAIL-Mu3, and were further assayed by sequencing.
Identification of enzyme-digested products of pET32a/TRAIL-Mu3. The pET32a plasmid and TRAIL-Mu3 DNA were digested by NdeI and EcoRI enzymes and detected by electrophoresis. The results demonstrated that there were two segments of $\sim 500 \mathrm{bp}$ and $\sim 5.4 \mathrm{~kb}$ (Fig. 1C), which were equal to the size of the TRAIL-Mu3 gene and pET32a plasmid, respectively. Subsequently, the plasmids were ligated, transformed into bacteria and cultured. A total of seven single colonies were isolated and the plasmids were extracted. The plasmids were digested and detected by agarose gel electrophoresis. The results demonstrated that there were two segments of $\sim 500$ bp and $\sim 5.4 \mathrm{~kb}$ in lines 2, 3, 4 and 5 (Fig. 1D), which were equal to the size of the TRAIL-Mu3 gene and pET32a plasmid, respectively. Therefore, these four colonies were transformed with pET32a/TRAIL-Mu3, and they were further analyzed by sequencing.

pET32a/TRAIL-Mu3 expression. As presented in Fig. 1E, there was strong expression in the prior-to-induction electrophoresis sample, post-electrophoresis sample, supernatant and precipitate, and that this was most apparent in the supernatant.

Purification of TRAIL-MU3 protein. The results of this analysis are presented in Fig. 2. Following cation exchange purification (Fig. 2A), $15 \mathrm{ml}$ eluent with a concentration of $2.273 \mathrm{mg} / \mathrm{ml}$ was obtained. The purity of TRAIL-Mu3 was thus demonstrated to be high. Via hydroxyapatite purification (Fig. 2B), $12 \mathrm{ml}$ hydroxyapatite eluent with a concentration of $2.080 \mathrm{mg} / \mathrm{ml}$ was obtained. The purpose of this step was to attempt to remove contaminating proteins and pyrogen. Following anion exchange purification (Fig. 2C) $20 \mathrm{ml}$ flow through fluid with a concentration of $0.846 \mathrm{mg} / \mathrm{ml}$ was obtained. In this step, the pyrogen was further removed. Following repeated purification, sufficient protein was obtained to evaluate its bioactivity in vitro and in vivo.

Western blot analysis of TRAIL-Mu3 protein. The results of the western blotting (Fig. 3) demonstrated that there was a positive band of $\sim 19.6 \mathrm{kDa}$ for TRAIL-Mu3 and the TRAIL 

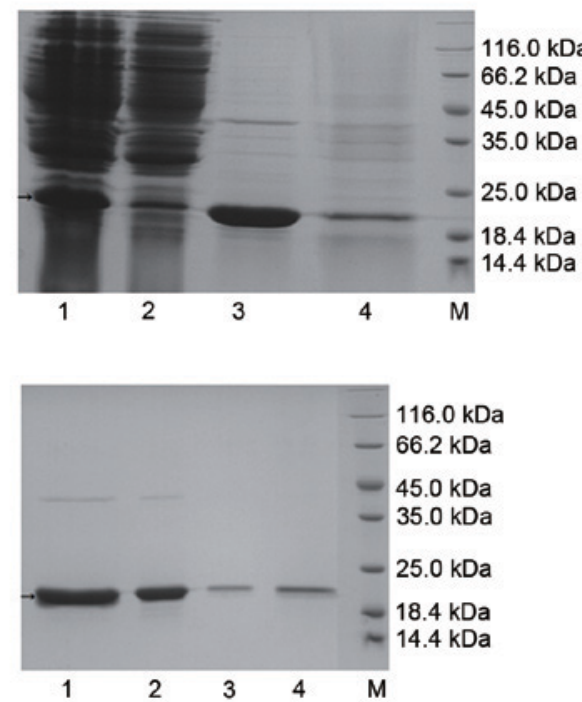

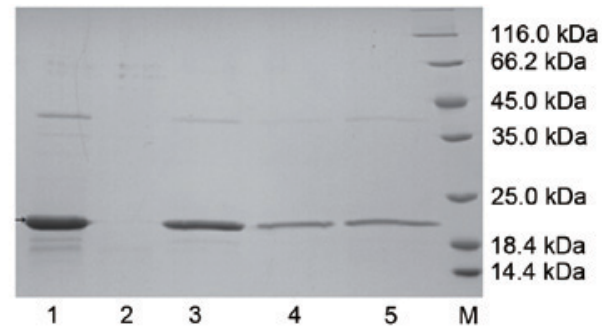

Figure 2. Purification of TRAIL-Mu3 protein. (A) Cation exchange purification. Lane 1, cation exchange liquid; lane 2, cation exchange flow-through liquid; lane 3, cation exchange eluate; lane 4, cation exchange sodium hydroxide eluent; $M$, unstained protein molecular weight marker. (B) Hydroxyapatite purification. Lane 1, hydroxyapatite sample solution; lane 2, hydroxyapatite flow-through liquid; lane 3 , hydroxyapatite sodium chloride eluent; lane 4 , hydroxyapatite phosphate eluent; M, unstained protein molecular weight marker. (C) Anion exchange purification. Lane 1, anion exchange liquid; lane 2, anion exchange flow-through liquid; lane 3, sodium chloride eluent; lane 4, sodium hydroxide eluent; M, unstained protein molecular weight marker. TRAIL, tumor necrosis factor ligand superfamily member 10 .

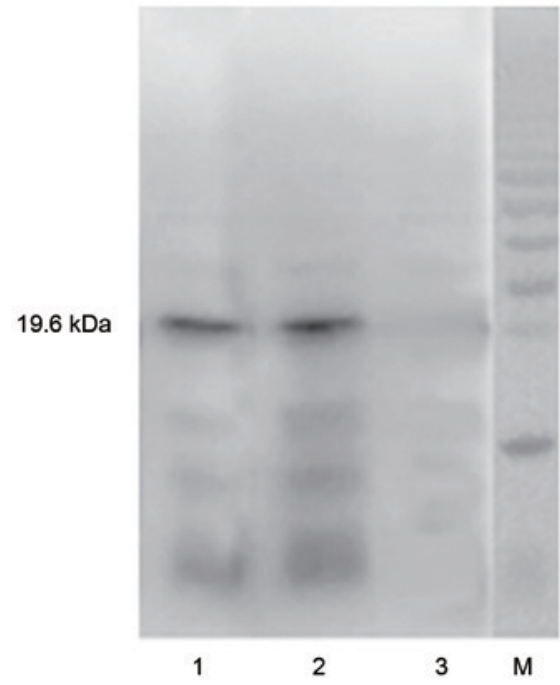

Figure 3. Western blot detection of TRAIL-Mu3 protein. Lane 1, TRAIL-Mu3; lane 2, TRAIL; lane 3, negative control; M: Thermo Fisher Scientific, Inc., page ruler pre-stained protein ladder. TRAIL, tumor necrosis factor ligand superfamily member 10 .

protein. However, the same band was not present for the supernatant of lysed BL21 (DE3) E. coli.

Immunofluorescence analysis. The results demonstrated that there was little TRAIL aggregated on the cell membrane of SW620 cells (Fig. 4A); however, extensive TRAIL-Mu3 was observed around the cell membrane of the cells (Fig. 4B).

TRAIL-Mu3 mediated toxicity on colorectal cancer cell lines. The $\mathrm{IC}_{50}$ values of TRAIL and TRAIL-Mu3 in various colorectal cancer cell lines are presented in Table I. The results demonstrated that TRAIL-Mu3 was able to enhance the antitumor effects of TRAIL in all five colorectal cancer cell lines. In addition, TRAIL-Mu3 was able to reverse the resistance of TRAIL-resistant HT-29 colorectal cancer cells.

Antitumor effects in HT-29 xenograft nude mice. The treatment commenced on day 10 following the injection of tumor cells. The tumor growth curves and inhibitive rates are presented in Fig. 5. The results demonstrated that the tumor growth inhibition rate increased with the increase in TRAIL-Mu3 concentration. In addition, the tumor growth inhibition rate of TRAIL-Mu3 was significantly increased compared with TRAIL at the same concentration of $45 \mathrm{mg} / \mathrm{kg}$.

Side effects, survival quality and analysis. All the mice in the TRAIL and TRAIL-Mu3 groups began to manifest slight syndromes such a stunt responses, bad appetite, and little activity on the 8th day after the treatment. However, there were no significant differences between TRAIL and TRAIL-Mu3 groups in mental status, weight, appetite and so on.

\section{Discussion}

TRAIL, which is able to selectively induce apoptosis in cancer cells, is a potential targeted drug for cancer therapy (20). However, its clinical use is limited by cellular resistance to cell death which occurs in $\sim 50 \%$ of cancer cells (21). Various methods have been developed to overcome TRAIL resistance $(4,22)$.

In recent years, CPPs have become one of the most popular techniques for intracellular access (6). CPPs are typically short cationic sequences and may be derived from natural sources or be synthetically designed constructs. It was previously demonstrated that this sequence was able to be shortened to a few amino acids, without altering its translocation capacity (23). In 
A

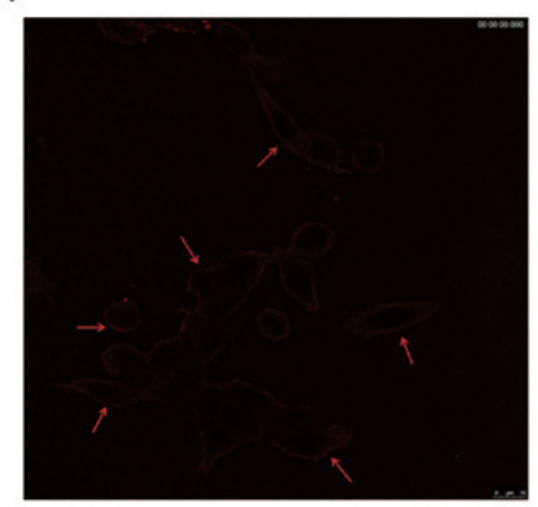

B

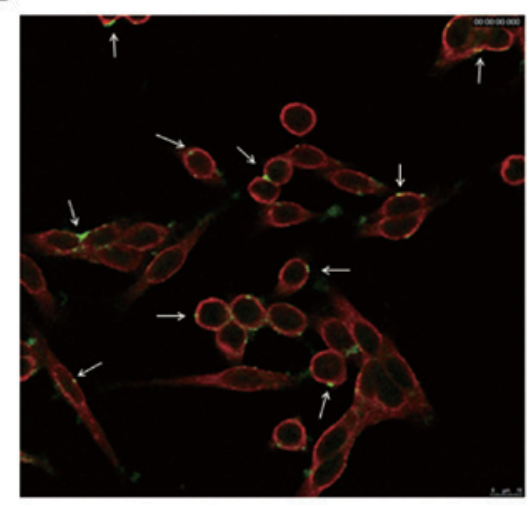

Figure 4. Immunofluorescence analysis (magnification x400). (A) SW620 cells treated with TRAIL (double staining of TRAIL and cell membrane; red arrows indicate cell membranes). (B) SW620 cells treated with TRAIL-Mu3 (double staining of TRAIL-Mu3 and cell membrane; white arrows indicate that stained TRAIL-Mu3 was aggregated around the cell surface). TRAIL, tumor necrosis factor ligand superfamily member 10.

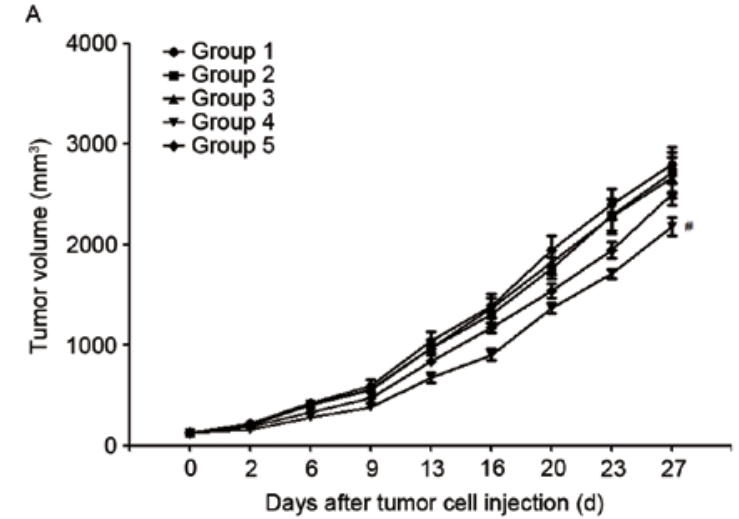

B

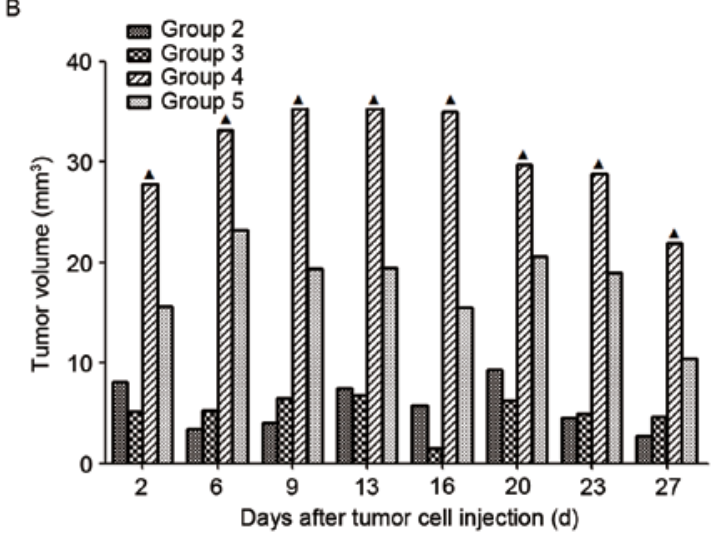

Figure 5. (A) Tumor growth curve and (B) inhibition rates. Group 1, treated with vehicle; Group 2, treated with TRAIL-Mu3 at a concentration of $5 \mathrm{mg} / \mathrm{kg}$; group 3, treated with TRAIL-Mu3 at a concentration of $15 \mathrm{mg} / \mathrm{kg}$; group 4, treated with TRAIL-Mu3 at a concentration of $45 \mathrm{mg} / \mathrm{kg}$; and group 5 , treated with TRAIL at a concentration of $45 \mathrm{mg} / \mathrm{kg}$. ${ }^{\#} \mathrm{P}<0.05$ vs. group $1,2,3,5 ;{ }^{\wedge} \mathrm{P}<0.05$ vs. group $2,3,5$. TRAIL, tumor necrosis factor ligand superfamily member 10 .

addition, this highly efficient translocation capacity has been observed in a variety of cell lines with minimal toxicity, overcoming challenges frequently associated with other delivery methods $(24,25)$.

In the present study, 5 amino acids of the extracellular region (114-281aa) of wild-type TRAIL were selectively altered to form a continuous sequence of $8 \mathrm{Arg}$ residues
Table I. The $\mathrm{IC}_{50}$ values of TRAIL and TRAIL-Mu3 in various colorectal cancer cell lines.

\begin{tabular}{lcc}
\hline & \multicolumn{2}{c}{$\mathrm{IC}_{50}, \mu \mathrm{g} / \mathrm{ml}$} \\
\cline { 2 - 3 } Cell line & TRAIL & TRAIL-Mu3 \\
\hline HCT-15 & 0.008 & 0.001 \\
COLO 205 & 0.008 & 0.002 \\
SW620 & 0.009 & 0.002 \\
HT-29 & $>100$ & 0.030 \\
HCT 116 & 0.015 & 0.002 \\
\hline
\end{tabular}

$\mathrm{IC}_{50}$, half-maximal inhibitory concentration; TRAIL, tumor necrosis factor ligand superfamily member 10 .

which approximately maintained the conformation of TRAIL protein, and additionally had a penetrating peptide-like structure. This TMPPA was termed TRAIL-Mu3.

Following PCR amplification with designed primers, a target gene of $\sim 500$ bp was obtained. The gene was ligated with pMD19-T vector and pET32a plasmid, successively. Following transformation into bacteria, culturing, enzyme digestion, electrophoresis and sequencing, it was confirmed that TRAIL-Mu3 was successfully synthesized. Positively-transformed bacteria with plasmid pET32a/TRAIL-Mu3 were obtained. The following SDS-PAGE electrophoresis demonstrated that the positively-transformed bacteria with plasmid pET32a/TRAIL-Mu3 were able to express TRAIL-Mu3 successfully. Subsequently, cation exchange purification, hydroxyapatite purification and anion exchange purification methods were used to purify the TRAIL-Mu3 proteins. The results demonstrated that the purity of TRAIL-Mu3 protein was high. Western blot analysis further confirmed that the TRAIL-Mu3 protein was successfully obtained.

TRAIL-Mu3 has a penetrating peptide-like structure; therefore, the affinity of TRAIL-Mu3 to the cancer cell membrane was detected. In the immunofluorescence analysis, it was observed that TRAIL-Mu3 exhibited significantly stronger affinity to the SW620 colorectal cancer cell 
membrane compared with TRAIL. It was hypothesized that TRAIL-Mu3 may enhance the affinity to the cancer cell membrane. TRAIL-Mu3 was able to aggregate on the cancer cell membrane, and exerted increased signal transduction and antitumor effects.

The present study aimed to investigate the antitumor effects of TRAIL-Mu3 on tumor cells in vitro and in vivo. The antitumor effects of TRAIL-Mu3 and TRAIL were detected in 32 different tumor cell lines through CCK-8 method. It was observed that TRAIL-Mu3 exhibited markedly stronger antitumor effects compared with TRAIL in these 32 tumor cell lines (data not shown), including 5 colorectal tumor cell lines. Additionally, TRAIL-Mu3 was able to reverse the resistance of TRAIL-resistant tumor cell lines (data not shown) including HT-29 tumor cell line. The antitumor effects of TRAIL-Mu3 were further detected in HT-29 tumor-bearing mice. Similarly, TRAIL-Mu3 at the same concentration exhibited significantly better antitumor effects compared with TRAIL on HT-29 tumor-bearing nude mice.

To the best of our knowledge, the present study was the first to introduce the concept of mutations in TRAIL and to obtain the novel drug TRAIL-Mu3. In addition, RAIL-Mu3 exerted stronger antitumor effects compared with TRAIL in colorectal cancer in vitro and in vivo. However, there remain certain limitations to the present study. The detailed mechanisms underlying the antitumor effects of TRAIL-Mu3 were not completely elucidated. Future studies are required to complete the investigation into the mechanism.

The present study constructed a novel drug TMPPA termed TRAIL-Mu3. TRAIL-Mu3 exerted significantly stronger antitumor effects compared with TRAIL on colorectal cancer in vitro and in vivo. TRAIL-Mu3 was able to reverse the resistance of TRAIL-resistant HT-29 colorectal cancer cells successfully.

\section{Acknowledgements}

The present study was supported by grants from the National Natural Scientific Foundation of China (grant nos. 81301962 and 81372444).

\section{References}

1. Fulda S: Safety and tolerability of TRAIL receptor agonists in cancer treatment. Eur J Clin Pharmacol 71: 525-527, 2015.

2. Wiley SR, Schooley K, Smolak PJ, Din WS, Huang CP, Nicholl JK, Sutherland GR, Smith TD, Rauch C, Smith CA, et al: Identification and characterization of a new member of the TNF family that induces apoptosis. Immunity 3: 673-682, 1995.

3. Ashkenazi A: Targeting the extrinsic apoptosis pathway in cancer. Cytokine Growth Factor Rev 19: 325-331, 2008.

4. Dimberg LY, Anderson CK, Camidge R, Behbakht K, Thorburn A and Ford HL: On the TRAIL to successful cancer therapy? Predicting and counteracting resistance against TRAIL-based therapeutics. Oncogene 32: 1341-1350, 2013.

5. Adamik P, Emmenegger T, Briedis M, Gustafsson L, Henshaw I, Krist M, Laaksonen T, Liechti F, Procházka P, Salewski V and Hahn S: Barrier crossing in small avian migrants: Individual tracking reveals prolonged nocturnal flights into the day as a common migratory strategy. Sci Rep 6: 21560, 2016.

6. Koren E and Torchilin VP: Cell-penetrating peptides: Breaking through to the other side. Trends Mol Med 18: 385-393, 2012

7. Wagstaff KM and Jans DA: Protein transduction: Cell penetrating peptides and their therapeutic applications. Curr Med Chem 13: $1371-1387,2006$
8. Lin W, Xie X, Yang Y, Fu X, Liu H and Deng J: Thermosensitive magnetic liposomes with doxorubicin cell-penetrating peptides conjugate for enhanced and targeted cancer therapy. Drug Deliv 23: 3436-3443, 2016.

9. Sugawara K, Shinohara H, Kadoya T and Kuramitz H: Sensing lymphoma cells based on a cell-penetrating/apoptosis-inducing/electron-transfer peptide probe. Anal Chim Acta 924: 106-113, 2016.

10. Yoo J, Lee D, Gujrati V, Rejinold NS, Lekshmi KM, Uthaman S, Jeong C, Park IK, Jon S and Kim YC: Bioreducible branched poly(modified nona-arginine) cell-penetrating peptide as a novel gene delivery platform. J Control Release 246: 142-154, 2017.

11. Zhu H, Li Z, Mao S, Ma B, Zhou S, Deng L, Liu T, Cui D, Zhao Y, He J, et al: Antitumor effect of sFlt-1 gene therapy system mediated by Bifidobacterium Infantis on Lewis lung cancer in mice. Cancer Gene Ther 18: 884-896, 2011.

12. Li ZJ, Zhu H, Ma BY, Zhao F, Mao SH, Liu TG, He JP, Deng LC, Yi C and Huang Y: Inhibitory effect of Bifidobacterium infantis-mediated sKDR prokaryotic expression system on angiogenesis and growth of Lewis lung cancer in mice. BMC Cancer 12: 155, 2012.

13. Gan HT, Lee J, Latiff SM, Chuah C, Toh P, Lee WY and Gagnon P: Characterization and removal of aggregates formed by nonspecific interaction of IgM monoclonal antibodies with chromatin catabolites during cell culture production. J Chromatogr A 1291: 33-40, 2013

14. Zeng H, Yuan Z, Zhu H, Li L, Shi H, Wang Z, Fan Y, Deng Q, Zeng J, He Y, et al: Expression of hPNAS-4 radiosensitizes Lewis lung cancer. Int J Radiat Oncol Biol Phys 84: e533-e540, 2012.

15. Zhu H, Zhang Y, Hu X, Yi C, Zhong S, Wang Y and Yang F: The effects of high-dose qinggan huoxue recipe on acute liver failure induced by d-galactosamine in rats. Evid Based Complement Alternat Med 2013: 905715, 2013.

16. Wang C, Yan X, Meng T, Hu T and Pan J: Immunofluorescence analysis of membrane-associated proteins for clathrin-mediated endocytosis in plant root cells. Methods Mol Biol 1662: 151-157, 2017.

17. Wang H, Li D, Hu Z, Zhao S, Zheng Z and Li W: Protective effects of green tea polyphenol against renal injury through ros-mediated JNK-MAPK pathway in lead exposed rats. Mol Cells 39: 508-513, 2016.

18. Zhu H, Zhao F, Yu S, He J, Deng L, Yi C and Huang Y: The synergistic effects of low-dose irinotecan and TRAIL on TRAIL-resistant HT-29 colon carcinoma in vitro and in vivo. Int J Mol Med 30: 1087-1094, 2012.

19. Zhu H, Huang M, Ren D, He J, Zhao F, Yi C and Huang Y: The synergistic effects of low dose fluorouracil and TRAIL on TRAIL-resistant human gastric adenocarcinoma AGS cells. Biomed Res Int 2013: 293874, 2013.

20. Cui DD, Huang Y, Mao SH, Chen SC, Qiu M, Ji LL and Yi C: Synergistic antitumor effect of TRAIL and adriamycin on the human breast cancer cell line MCF-7. Braz J Med Biol Res 42: 854-862, 2009.

21. Zhang X, Zhao J, Zhu W, Gou H, Cao D, Yang Y, Huang Y and Yi C: Synergistic effect of subtoxic-dose cisplatin and TRAIL to mediate apoptosis by down-regulating Decoy receptor 2 and up-regulating caspase-8, caspase- 9 and bax expression on NCI-H460 and A549 cells. Iran J Basic Med Sci 16: 710-718, 2013.

22. Jiang $\mathrm{Q}$, Zhu H, Liang B, Huang Y and Li C: Apoptosis-inducing effect of the DR5 monoclonal antibody, D-6, alone or in combination with cisplatin, on A2780 ovarian cancer cells. Mol Med Rep 6: 316-320, 2012.

23. Falanga A, Galdiero M and Galdiero S: Membranotropic cell penetrating peptides: The outstanding Journey. Int J Mol Sci 16: 25323-25337, 2015.

24. Xie X, Yang Y, Lin W, Liu H, Liu H, Yang Y, Chen Y, Fu X and Deng J: Cell-penetrating peptide-siRNA conjugate loaded YSA-modified nanobubbles for ultrasound triggered siRNA delivery. Colloids Surf B Biointerfaces 136: 641-650, 2015.

25. Parnaste L, Arukuusk P, Zagato E, Braeckmans K and Langel U: Methods to follow intracellular trafficking of cell-penetrating peptides. J Drug Target 24: 508-519, 2016. 